\title{
Discriminating Single-Photon States Unambiguously in High Dimensions
}

\author{
Megan Agnew, ${ }^{1,2}$ Eliot Bolduc, ${ }^{2}$ Kevin J. Resch, ${ }^{1}$ Sonja Franke-Arnold, ${ }^{3}$ and Jonathan Leach ${ }^{2}$ \\ ${ }^{1}$ Department of Physics \& Astronomy and Institute for Quantum Computing, University of Waterloo, Waterloo N2L 3G1, Canada \\ ${ }^{2}$ SUPA, School of Engineering and Physical Sciences, Heriot-Watt University, Edinburgh EH14 4AS, United Kingdom \\ ${ }^{3}$ SUPA, School of Physics and Astronomy, University of Glasgow, Glasgow G12 8QQ, United Kingdom
}

(Received 14 March 2014; published 7 July 2014)

\begin{abstract}
The ability to uniquely identify a quantum state is integral to quantum science, but for nonorthogonal states, quantum mechanics precludes deterministic, error-free discrimination. However, using the nondeterministic protocol of unambiguous state discrimination enables the error-free differentiation of states, at the cost of a lower frequency of success. We discriminate experimentally between nonorthogonal, highdimensional states encoded in single photons; our results range from dimension $d=2$ to $d=14$. We quantify the performance of our method by comparing the total measured error rate to the theoretical rate predicted by minimum-error state discrimination. For the chosen states, we find a lower error rate by more than 1 standard deviation for dimensions up to $d=12$. This method will find immediate application in high-dimensional implementations of quantum information protocols, such as quantum cryptography.
\end{abstract}

DOI: 10.1103/PhysRevLett.113.020501

Discriminating between different quantum states without error is a fundamental requirement of quantum information science. However, due to the nature of quantum mechanics, only orthogonal states can be exactly discriminated without error $100 \%$ of the time. In contrast, the discrimination of nonorthogonal states requires a decrease in either detection accuracy, using minimum-error state discrimination, or detection frequency, using unambiguous state discrimination. Minimum-error state discrimination (MESD) always provides information about the state, although the information may be incorrect [1]. Conversely, unambiguous state discrimination (USD) provides either the correct information about a detected state or inconclusive information about the state [2-15].

High-dimensional quantum states are an important resource for quantum information. In comparison to qubits, the use of qudits, which are states belonging to a $d$-dimensional space, provides access to a larger alphabet and correspondingly higher information rates and a higher tolerance to noise. The ability to unambiguously discriminate such states is thus of key importance, and successful protocols that accomplish this task will extend the use of these states in quantum information science. Examples of such systems include the time degree of freedom and the spatial light profile, or more specifically the orbital angular momentum (OAM) degree of freedom, which we use in this work [16-29]. High-dimensional USD is also potentially relevant for pattern recognition in quantum and classical regimes, as images typically contain very large numbers of spatial modes and are nonorthogonal to one another [30].

Published by the American Physical Society under the terms of the Creative Commons Attribution 3.0 License. Further distribution of this work must maintain attribution to the author(s) and the published article's title, journal citation, and DOI.
PACS numbers: 03.67.Mn, 03.65.Ta, 42.50.Dv, 42.50.Ex

The problem of unambiguous discrimination of qudit states has received a great deal of attention [31-36]. USD was first experimentally realized, with a classical light source, to distinguish two nonorthogonal states in the polarization degree of freedom [37]. A subsequent experiment with a similar source extended this to distinguish three states encoded in three-dimensional photon path information [38]. USD has also been performed for two mixed polarization states using a quantum dot single-photon source [39].

In this Letter, we discriminate unambiguously between nonorthogonal quantum states encoded in single photons, in dimensions ranging from $d=2$ to $d=14$. While USD theoretically promises the unambiguous discrimination of any set of states, real experimental situations always include error sources, and perfect discrimination in an experimental environment is challenging. Even with these unavoidable errors, we show that our scheme successfully discriminates between the chosen states and does so with lower error rates than those predicted by MESD. We note that here we implement USD as a sequential measurement of all required detection states. Using instead simultaneous detection, e.g., detection based on OAM sorter technology [40,41], would allow unambiguous discrimination at the single-photon level.

To perfectly distinguish orthogonal states, one requires projections onto the orthogonal state basis, giving $d$ measurement outcomes in a $d$-dimensional space. To implement the USD protocol, which distinguishes nonorthogonal states, one requires the introduction of an additional measurement outcome-an inconclusive result -into the procedure, providing $d+1$ measurement possibilities. One way to increase the number of measurement outcomes is by introducing an ancillary dimension or degree of freedom; orbital angular momentum lends itself well to this treatment, as it provides an unlimited supply of additional dimensions. The introduction of the inconclusive 
result enables the remaining measurement outcomes to be orthogonalized [42]. The protocol then provides one of the following: a correct state identification, in which case the state is known with certainty, or an inconclusive result, in which case no information is known about the state.

In this Letter, we choose $d$ states in $d$ dimensions that have an equal overlap with each other; these are referred to as equally probable, linearly independent, symmetrical states and, compared to less symmetric states, have a maximal discrimination probability $[5,43]$. See Fig. 1(a) for an example in three dimensions. Note that all of these states have only real amplitudes. The overlap between any two states is then a function of the parameter $\theta$, given by

$$
\left\langle\Psi_{i} \mid \Psi_{j}\right\rangle=\frac{d \cos ^{2} \theta-1}{d-1},
$$

for $i \neq j$. To ensure positive overlap between the input states, the maximum value of $\theta$ is $\theta_{\max }=\cos ^{-1} \sqrt{1 / d}$ [43].

In the problem of USD, we must establish a set of measurement states $\left\{\left|D_{i}\right\rangle\right\}$ to distinguish the set of input states $\left\{\left|\Psi_{i}\right\rangle\right\}$. To achieve this, for every state $\left|\Psi_{i}\right\rangle$, we first identify a preliminary measurement state $\left|\Psi_{i}^{\perp}\right\rangle$; this preliminary state is orthogonal to all other states $\left|\Psi_{j}\right\rangle$ (for $j \neq i$ ) but has a nonzero overlap with $\left|\Psi_{i}\right\rangle$. Because of this definition, a detection with $\left|\Psi_{i}^{\perp}\right\rangle\left\langle\Psi_{i}^{\perp}\right|$ will unambiguously indicate that the photon was in state $\left|\Psi_{i}\right\rangle$. These $d$ preliminary measurement states $\left\{\left|\Psi_{i}^{\perp}\right\rangle\right\}$, however, do not generally form an orthonormal basis set. This can be achieved by extending the preliminary measurement states to an ancillary dimension, followed by normalization to obtain $d$ measurement states $\left\{\left|D_{i}\right\rangle\right\}$. The basis set is completed by including an additional state $\left|D_{d+1}\right\rangle$ orthogonal to all other measurement states, so that the whole $(d+1)$-dimensional basis of measurement states is $\left\{\left|D_{i}\right\rangle\right\}$ with $\left\langle D_{i} \mid D_{j}\right\rangle=\delta_{i j}$.

The probability of obtaining an inconclusive result $\left|\left\langle\Psi_{i} \mid D_{d+1}\right\rangle\right|^{2}$ and the probability of correctly identifying a state $\left|\left\langle\Psi_{i} \mid D_{i}\right\rangle\right|^{2}$ sum to unity, as the probability of an error is by definition 0 . The probability of an inconclusive result is precisely the overlap between any two input states $[3,5]$.

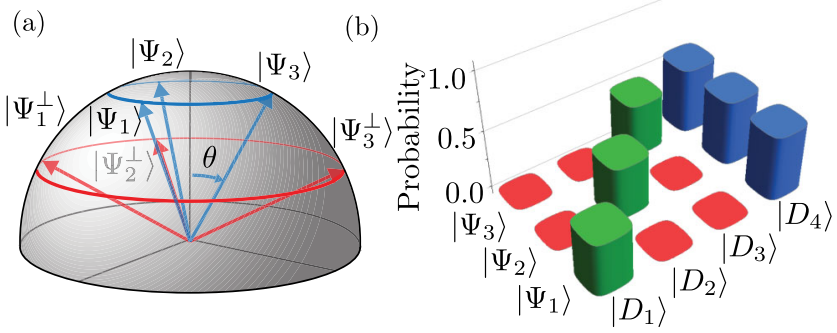

FIG. 1 (color online). Vectors and probabilities in three dimensions. (a) As the states we consider have real amplitudes, they can be represented on a sphere whose axes are the amplitudes of each basis vector. The vectors we choose to discriminate in dimension $d=3$, $\left\{\left|\Psi_{i}\right\rangle\right\}$ with $\theta \approx 33^{\circ}$, are shown in blue. Vectors perpendicular to each pair $\left(\left\{\left|\Psi_{i}^{\perp}\right\rangle\right\}\right)$ are shown in red. (b) Theoretically calculated probabilities of discrimination for the vectors shown in (a).
Thus, using Eq. (1), we can write the probabilities of successful identification, erroneous identification, and inconclusive result as

$$
\begin{aligned}
& p_{\text {suc }}=\frac{d}{d-1} \sin ^{2} \theta, \\
& p_{\text {err }}=0, \\
& p_{\text {inc }}=\frac{d \cos ^{2} \theta-1}{d-1} .
\end{aligned}
$$

Theoretical predictions of these values for states in three dimensions are shown in Fig. 1(b).

We use the process outlined above to find the discrimination states for a range of input states in a range of dimensions, and we use them to implement USD as a sequential measurement on orbital angular momentum states. Our experimental procedure is as follows. We produce entangled photons by spontaneous parametric down-conversion [44] in a 3-mm type-I $\beta$-barium borate (BBO) crystal with a phase mismatch factor of approximately $\phi=-1$. We pump the crystal with a $100-\mathrm{mW}$ laser at $405 \mathrm{~nm}$. In each path, we image the plane of the BBO crystal to a different section of a spatial light modulator (SLM), allowing us to manipulate both the phase and the amplitude of each photon's mode with high fidelity. The simplified experimental setup is shown in Fig. 2.

The photons produced from the BBO crystal are entangled in their orbital angular momentum in the two-photon state $|\psi\rangle=\sum_{\ell=-\infty}^{\infty} c_{\ell}|\ell\rangle_{A} \otimes|-\ell\rangle_{B}$, where $\left|c_{\ell}\right|^{2}$ is the probability of finding photon $A$ with OAM $\ell \hbar$ and photon $B$ with OAM $-\ell \hbar$ [45]. The SLM in our experiment performs a number of functions in regard to this state: first, it allows us to select a range of OAM values and explore a discrete dimension space, and second, it allows us to equalize the probabilities of detection, a process similar to entanglement concentration [46]. Finally, in a procedure similar to that in Refs. [21-23,26-28], the SLM, in combination with the single-mode fibers, allows us to perform high-fidelity projective measurements on the signal and idler photons. By displaying a hologram of the conjugate phase of a particular mode, we convert the chosen state into the Gaussian mode, which can then be coupled into a single-mode fiber.

The entanglement of the OAM degree of freedom allows the use of remote state preparation $[47,48]$, which enables us to herald the presence of a range of single-photon states $\left|\Psi_{i}\right\rangle$. These heralded states are prepared by using one-half of the SLM in combination with a single-mode fiber. Consequently, the detection of a single photon in the first arm collapses the photon in the other arm into the desired state. The second path is then used to perform the state discrimination measurements $\left|D_{j}\right\rangle$ on the heralded state $\left|\Psi_{i}\right\rangle$, and we measure the coincidences between the two paths. In our experiment, as we use a single SLM, the measurements on the signal and idler modes are performed simultaneously. In this manner, the input states are prepared 
Sample holograms for state preparation
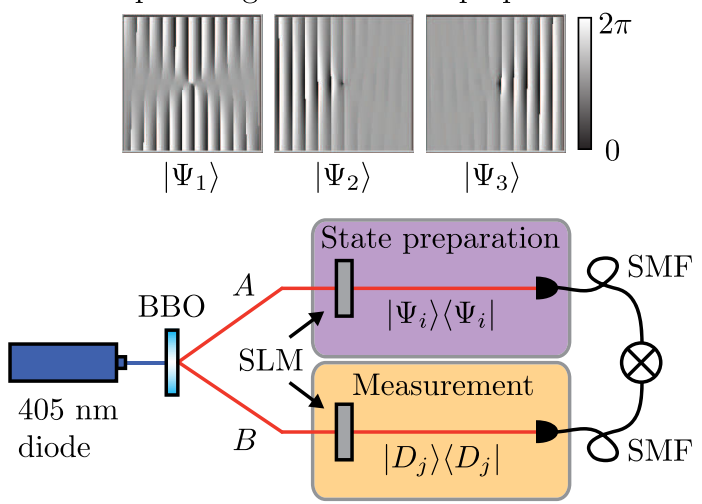

Sample holograms for measurement
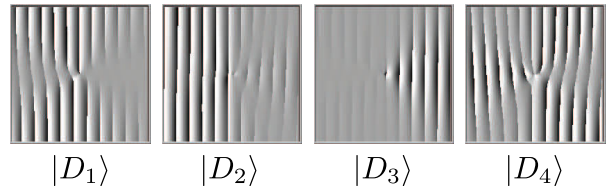

FIG. 2 (color online). Experimental setup. A pair of entangled photons is produced in a BBO crystal. Arm $A$ is used to prepare a state $\left|\Psi_{i}\right\rangle$, indicated by the purple box; arm $B$ is used to perform a measurement $\left|D_{j}\right\rangle$, indicated by the orange box. Each measurement is accomplished using an SLM and a single-mode fiber (SMF). The holograms shown are representative of those used for state preparation and measurement in dimension $d=3$.

at the same time as they are measured. However, identical results would be obtained using two spatially separated SLMs, where the preparation and measurement stages are performed one after another. Our chosen method of measurement does not limit the use of such states for tasks in quantum information processing.

For a given dimension $d$, we measure all $d+1$ measurement outcomes for each input state $\left|\Psi_{i}\right\rangle$. We use our measurements to calculate a quantity called the quantum contrast, which is defined by the coincidence rates normalized by the singles $Q_{i j}=C_{i j} /\left(S_{A i} S_{B j} t\right)$; this accounts for any variations in the quantum efficiency of the detection and generation of particular states. Here, $C_{i j}$ is the number of coincidence counts defined by an event in both detectors within a time window of $t=25 \mathrm{~ns}$. The quantities $S_{A i}$ and $S_{B j}$ represent the number of counts in path $A$ (heralding the preparation of $\left|\Psi_{i}\right\rangle$ ) and $B$ (measuring $\left|D_{j}\right\rangle$ ), respectively. We normalize this quantum contrast into probabilities using $P_{i j}=\left(Q_{i j}-1\right) / \sum_{j}\left(Q_{i j}-1\right)$. The -1 term accounts for the fact that two independent and uncorrelated sources will have a quantum contrast equal to unity. An integration time of $30 \mathrm{~s}$ was used for each measurement, and the maximal coincidence count rate was approximately $350 \mathrm{~Hz}$.

We have implemented our procedure for unambiguous discrimination of states in high dimensions ranging from $d=2$ to $d=14$ and with varying overlap between the states. In Fig. 3, we show the unambiguous discrimination of six states in $d=6$ dimensions.

Figure 3(a) shows the results at $\theta=40^{\circ}$ of measuring all $\left\{\left|\Psi_{i}\right\rangle\right\}$ states using all $\left\{\left|D_{j}\right\rangle\right\}$ measurements. The green
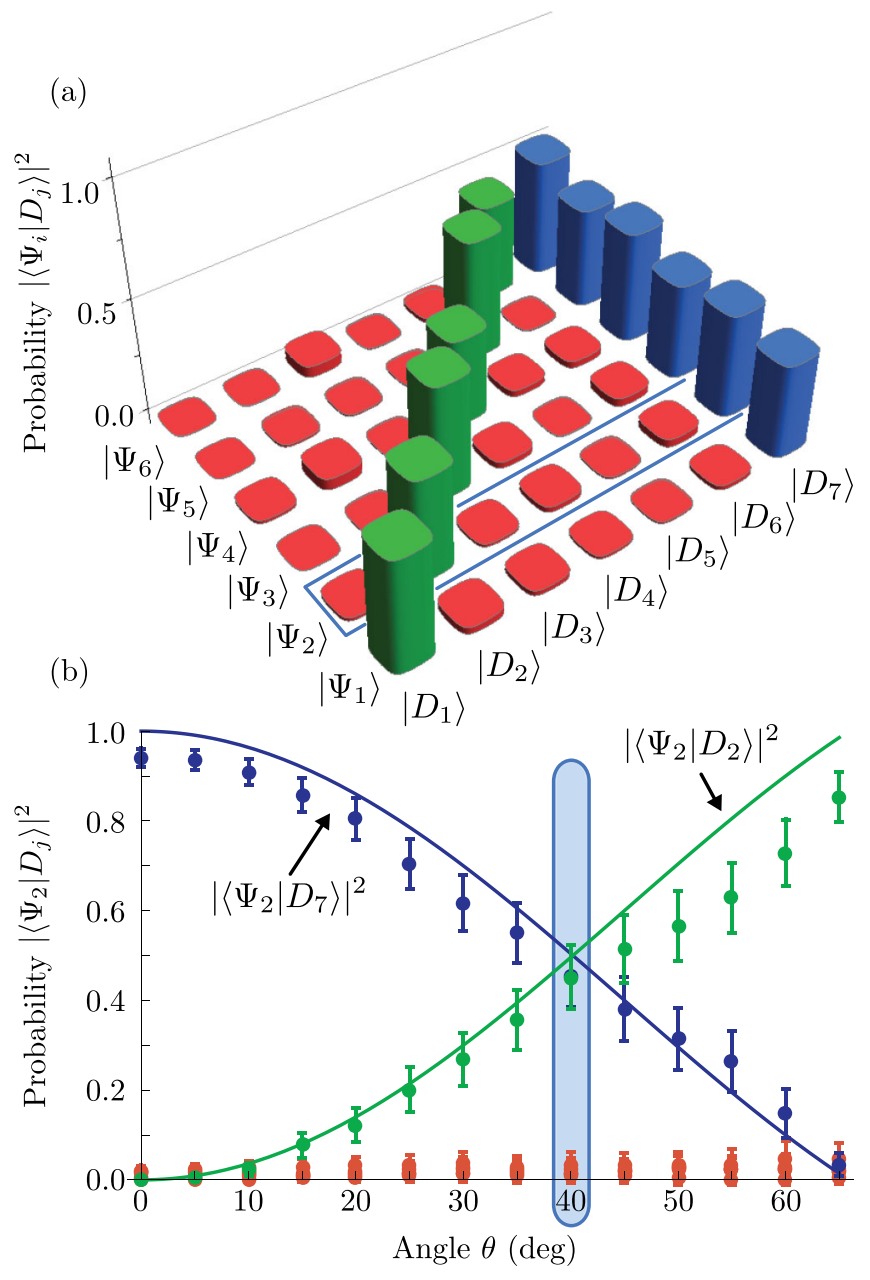

FIG. 3 (color online). Experimental results for dimension $d=6$. (a) Probabilities of detecting input states $\left|\Psi_{i}\right\rangle$ using detection states $\left|D_{j}\right\rangle$ when $\theta=40^{\circ}$. (b) Probabilities as a function of $\theta$ of identifying the state $\left|\Psi_{2}\right\rangle$ correctly (green points, $\left|D_{2}\right\rangle$ ), incorrectly (red points, $\left|D_{1}\right\rangle,\left|D_{3}\right\rangle,\left|D_{4}\right\rangle,\left|D_{5}\right\rangle,\left|D_{6}\right\rangle$ ), or inconclusively (blue points, $\left|D_{7}\right\rangle$ ). The points represent experimental data, while the solid lines represent theoretical values calculated using Eq. (2). The points within the shaded area in (b) correspond to the blue outlined box in (a). The uncertainties were calculated using Gaussian error propagation, where the measured counts $N$ were assumed to have a standard deviation of $\sqrt{N}$.

bars denote successful identifications, the red bars denote erroneous identifications, and the blue bars denote inconclusive results. As the probabilities of successful identification greatly exceed the probabilities of erroneous identification, it follows that each input state $\left|\Psi_{i}\right\rangle$ almost always results in either correct detection by $\left|D_{i}\right\rangle$ or the inconclusive outcome $\left|D_{7}\right\rangle$.

Figure 3(b) shows the results of measuring a specific state, in this case, $\left|\Psi_{2}\right\rangle$, using all $\left\{\left|D_{j}\right\rangle\right\}$ measurements, for a range of angles $\theta$. Each angle corresponds to a different overlap between the $\left\{\left|\Psi_{i}\right\rangle\right\}$ states, as in Eq. (1). An angle of $0^{\circ}$ corresponds to a complete overlap between the states and hence a completely inconclusive result; the probability for correct identification increases with $\theta$, with, in principle, 
perfect identification at $\theta \approx 66^{\circ}$. The solid lines indicate theoretical predictions from Eq. (2); our experimental data are in good agreement with these predictions.

While USD has the theoretical advantage of never misidentifying a state, in practice this is not possible to achieve. In experimental implementations, errors necessarily occur due to finite detector efficiency and errors caused by transformation optics. To evaluate the performance of our measurements, we compare our experimentally recorded errors to those theoretically predicted for the MESD protocol. A significant advantage is found in the case that the recorded errors for our scheme are smaller than those produced in MESD.

Because of the equal overlap between our input states, the minimum error rate for MESD in $d$ dimensions $[43,49]$ reduces to

$$
p_{\text {err }} \geq \frac{1}{2}\left(1-\sqrt{1-\left|\left\langle\Psi_{i} \mid \Psi_{j}\right\rangle\right|^{2}}\right),
$$

where the overlap $\left\langle\Psi_{i} \mid \Psi_{j}\right\rangle$ is given by Eq. (1). A violation of this inequality indicates that USD provides less ambiguity in state identification than is theoretically possible using MESD.

In Fig. 4, we compare this bound to the mean total error rate observed using our method. To determine our error rate, we first determine the error rate for a single input state $\left|\Psi_{i}\right\rangle$; this is the sum of all possible incorrect state identifications. We then average over all input states $\left\{\left|\Psi_{i}\right\rangle\right\}$ to obtain the mean total error rate.

Figure 4(a) shows the total error rate as a function of angle for the $d=6$ case. The total error rate for angles up to $\theta=30^{\circ}$ is at least 1 standard deviation below the MESD bound, demonstrating that our approach is particularly successful for states with large overlap. The total error rate exceeds the MESD bound at higher angles, where the states have lower overlap and are closer to orthogonal. In this case, the bound converges to 0 , matching the theoretical prediction for USD. Since the two schemes converge, it is inevitable that the experimentally measured errors exceed the ideal MESD curve at a sufficiently high angle.

Figure 4(b) shows the total error rate as a function of dimension for a fixed overlap of $1 / \sqrt{2}$ between the initial states. We choose a constant overlap so that the MESD bound is equal in all dimensions [in this case, $(1-\sqrt{1 / 2}) / 2 \approx 0.146]$. To achieve the constant overlap, the parameter $\theta$ must change with dimension [43]. The total error rate for dimensions up to $d=12$ is below the MESD bound by at least 1 standard deviation.

In dimensions $d \geq 13$, the bound for MESD is successfully violated, but by less than 1 standard deviation. This is due to two main factors. First, for all of these data, the average measured probability of obtaining an error, i.e., measuring a state $\left|\Psi_{i}\right\rangle$ with an incorrect detection state $\left|D_{j}\right\rangle$ $(i \notin\{j, d+1\})$, is approximately $1 \%$. As the dimension increases, so too does the number of opportunities to misidentify a state. Thus, the total error grows accordingly,
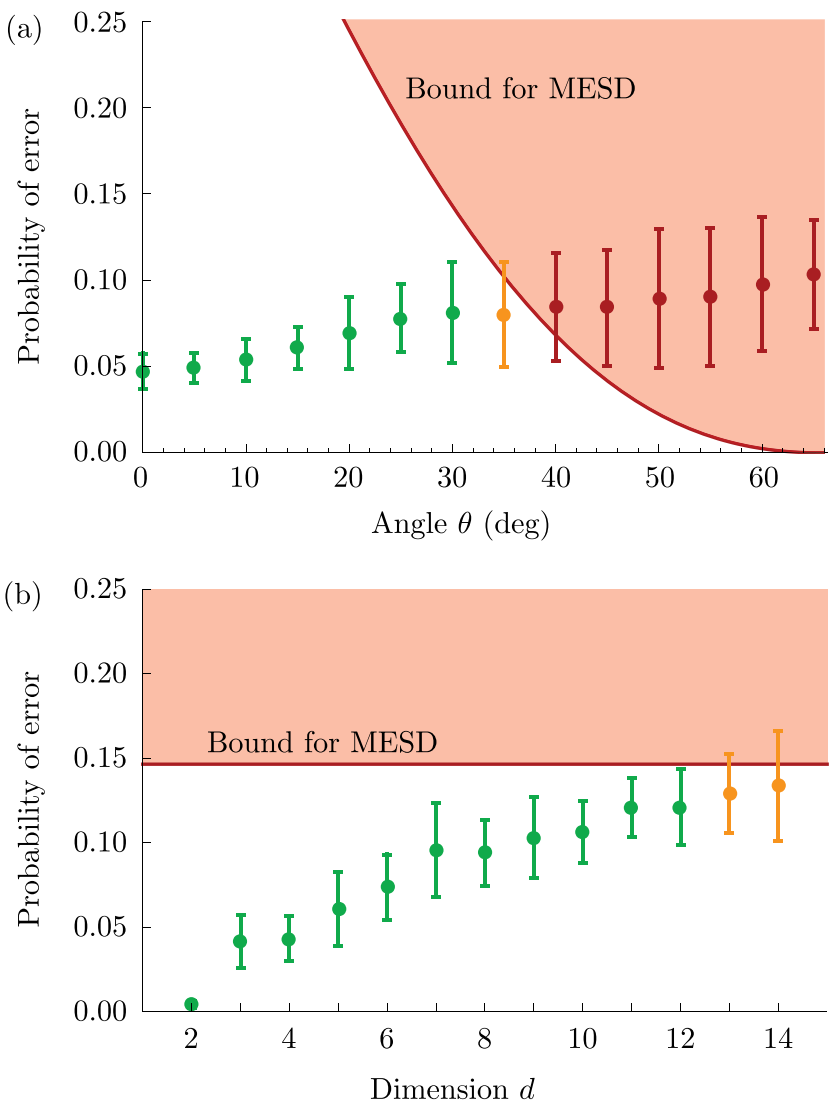

FIG. 4 (color online). Probability of error. (a) Mean total error rate as a function of angle in dimension $d=6$. (b) Mean total error rate as a function of dimension. Here, the angle $\theta$ is chosen individually for each dimension such that the MESD bound is the same in all dimensions. In both plots, the red line indicates the theoretical minimum error rate predicted for MESD. The green points denote error rates at least 1 standard deviation below this limit, the orange points denote error rates whose uncertainties extend above the limit, and the red points denote error rates above the limit. The uncertainties are the standard deviations associated with the mean values.

making it increasingly difficult to obtain a low total error. Second, due to the limited spiral bandwidth in the downconverted state, the probability amplitudes of the individual OAM modes decrease as $\ell$ increases. This limits the coincidence rate, and thus increases the uncertainty of the measurements, for high dimensions.

We have demonstrated USD via sequential measurements to distinguish $d$ nonorthogonal single-photon states in $d$-dimensional Hilbert spaces. In a modified setup, our method could be realized as a true positive operator-valued measure (POVM) experiment in high dimensions. To implement simultaneous measurement of the input states, one would require a scheme that takes any of the $d$ nonorthogonal states and produces $d+1$ orthogonal outputs. Such a scheme is similar in concept to current mode sorting technology that can, in principle, take $d$ orthogonal OAM input states and sort them into $d$ orthogonal transverse states [40,41]. Before using an OAM mode sorter, we 
would require an initial stage to convert the input states into a $(d+1)$-dimensional basis. Such an experimental implementation will be the focus of future work.

In our experiment, while experimental constraints prevent completely error-free identification, we have shown that, for a range of high-dimensional states, our method still provides a lower error rate than minimum-error state discrimination. With suitable improvements in SLM resolution, spiral bandwidth production, and detector efficiency, this could be increased to even higher dimensions. This method of state discrimination will allow the use of high-dimensional nonorthogonal states in quantum protocols, enabling secure quantum communication with larger alphabets.

We thank Sarah Croke for valuable discussions regarding this work. This work was supported by the Engineering and Physical Sciences Research Council (EPSRC). M. A. acknowledges financial support from the Natural Sciences and Engineering Research Council of Canada (NSERC).

[1] C. W. Helstrom, Quantum Detection and Estimation Theory (Academic, New York, 1976).

[2] I. D. Ivanovic, Phys. Lett. A 123, 257 (1987).

[3] D. Dieks, Phys. Lett. A 126, 303 (1988).

[4] A. Peres, Phys. Lett. A 128, 19 (1988).

[5] A. Chefles and S. M. Barnett, Phys. Lett. A 250, 223 (1998).

[6] A. Peres and D. R. Terno, J. Phys. A 31, 7105 (1998).

[7] Y. Sun, M. Hillery, and J. A. Bergou, Phys. Rev. A 64, 022311 (2001).

[8] T. Rudolph, R. W. Spekkens, and P. S. Turner, Phys. Rev. A 68, 010301 (2003).

[9] P. Raynal and N. Lütkenhaus, Phys. Rev. A 72, 022342 (2005).

[10] P. Raynal and N. Lütkenhaus, Phys. Rev. A 76, 052322 (2007).

[11] M. A. Jafarizadeh, M. Rezaei, N. Karimi, and A. R. Amiri, Phys. Rev. A 77, 042314 (2008).

[12] H. Sugimoto, T. Hashimoto, M. Horibe, and A. Hayashi, Phys. Rev. A 82, 032338 (2010).

[13] G. Waldherr, A. C. Dada, P. Neumann, F. Jelezko, E. Andersson, and J. Wrachtrup, Phys. Rev. Lett. 109, 180501 (2012).

[14] T. Zhou, Quantum Inf. Process. 11, 1669 (2012).

[15] J. Bergou, E. Feldman, and M. Hillery, Phys. Rev. Lett. 111, 100501 (2013).

[16] A. Mair, A. Vaziri, G. Weihs, and A. Zeilinger, Nature (London) 412, 313 (2001).

[17] S. P. Walborn, D. S. Lemelle, M. P. Almeida, and P. H. SoutoRibeiro, Phys. Rev. Lett. 96, 090501 (2006).

[18] J. B. Pors, A. Aiello, S. S. R. Oemrawsingh, M. P. van Exter, E. R. Eliel, and J. P. Woerdman, Phys. Rev. A 77, 033845 (2008).

[19] C. E. R. Souza, C. V. S. Borges, A. Z. Khoury, J. A. O. Huguenin, L. Aolita, and S. P. Walborn, Phys. Rev. A 77, 032345 (2008).

[20] S. P. Walborn, D. S. Lemelle, D. S. Tasca, and P. H. SoutoRibeiro, Phys. Rev. A 77, 062323 (2008).
[21] J. Leach, B. Jack, J. Romero, A. K. Jha, A. M. Yao, S. Franke-Arnold, D. G. Ireland, R. W. Boyd, S. M. Barnett, and M. J. Padgett, Science 329, 662 (2010).

[22] M. Agnew, J. Leach, M. McLaren, F. S. Roux, and R. W. Boyd, Phys. Rev. A 84, 062101 (2011).

[23] A. C. Dada, J. Leach, G. S. Buller, M. J. Padgett, and E. Andersson, Nat. Phys. 7, 677 (2011).

[24] L. E. Oxman and A. Z. Khoury, Phys. Rev. Lett. 106, 240503 (2011).

[25] B.-J. Pors, F. Miatto, G. W. 't Hooft, E. R. Eliel, and J. P. Woerdman, J. Opt. 13, 064008 (2011).

[26] M. Agnew, J. Leach, and R. Boyd, Eur. Phys. J. D 66, 1 (2012).

[27] J. Leach, E. Bolduc, D. J. Gauthier, and R. W. Boyd, Phys. Rev. A 85, 060304 (2012).

[28] M. Agnew, J. Z. Salvail, J. Leach, and R. W. Boyd, Phys. Rev. Lett. 111, 030402 (2013).

[29] J. M. Donohue, M. Agnew, J. Lavoie, and K. J. Resch, Phys. Rev. Lett. 111, 153602 (2013).

[30] M. Malik, O. S. Magaña-Loaiza, and R. W. Boyd, Appl. Phys. Lett. 101, 241103 (2012).

[31] U. Herzog and J.A. Bergou, Phys. Rev. A 78, 032320 (2008).

[32] S. Pang and S. Wu, Phys. Rev. A 80, 052320 (2009).

[33] J. A. Bergou, U. Futschik, and E. Feldman, Phys. Rev. Lett. 108, 250502 (2012).

[34] L. Chen and H. Lu, Sci. China Phys. Mech. Astron. 55, 55 (2012).

[35] L. Li, Phys. Rev. A 86, 032320 (2012).

[36] S. Franke-Arnold and J. Jeffers, Eur. Phys. J. D 66, 1 (2012)

[37] R. B. M. Clarke, A. Chefles, S. M. Barnett, and E. Riis, Phys. Rev. A 63, 040305(R) (2001).

[38] M. Mohseni, A. M. Steinberg, and J. A. Bergou, Phys. Rev. Lett. 93, 200403 (2004).

[39] G. A. Steudle, S. Knauer, U. Herzog, E. Stock, V. A. Haisler, D. Bimberg, and O. Benson, Phys. Rev. A 83, 050304 (2011).

[40] G. C. G. Berkhout, M. P. J. Lavery, J. Courtial, M. W. Beijersbergen, and M. J. Padgett, Phys. Rev. Lett. 105, 153601 (2010).

[41] M. Mirhosseini, M. Malik, Z. Shi, and R. W. Boyd, Nat. Commun. 4, 2781 (2013).

[42] M. A. Neumark, C.R. (Dokl.) Acad. Sci. URSS 41, 359 (1943).

[43] See Supplemental Material at http://link.aps.org/supplemental/ 10.1103/PhysRevLett.113.020501 for further information.

[44] S. P. Walborn, A. N. de Oliveira, R. S. Thebaldi, and C. H. Monken, Phys. Rev. A 69, 023811 (2004).

[45] J. P. Torres, A. Alexandrescu, and L. Torner, Phys. Rev. A 68, 050301 (2003).

[46] C. H. Bennett, H. J. Bernstein, S. Popescu, and B. Schumacher, Phys. Rev. A 53, 2046 (1996).

[47] C. H. Bennett, D. P. DiVincenzo, P. W. Shor, J. A. Smolin, B. M. Terhal, and W. K. Wootters, Phys. Rev. Lett. 87, 077902 (2001).

[48] W.-T. Liu, W. Wu, B.-Q. Ou, P.-X. Chen, C.-Z. Li, and J.-M. Yuan, Phys. Rev. A 76, 022308 (2007).

[49] D. Qiu, Phys. Rev. A 77, 012328 (2008). 